\title{
Modelling anomalous lensed quasars
}

\author{
Nicholas Bate* \\ The University of Melbourne \\ E-mail: nbate@physics.unimelb.edu.au

\section{Rachel Webster} \\ The University of Melbourne \\ E-mail: rwebster@physics.unimelb.edu.au
}

\section{Stuart Wyithe}

The University of Melbourne

E-mail: swyithe@physics.unimelb.edu.au

\begin{abstract}
Several gravitationally lensed quasars are observed with anomalous magnifications in pairs of images that straddle a critical curve. Simple theoretical arguments suggest that the magnification of these images should be approximately equivalent, whereas one image is observed to be significantly demagnified. Microlensing offers a possible explanation for this discrepancy. I will discuss the significance of the two key parameters in modelling this effect: the fraction of smooth matter in the lens at the image positions, and the size of the quasar emission region. We have applied our model to the anomalous lensed quasar MG 0414+0534, and found a 95 percent upper limit of $2.62 \times 10^{16} \mathrm{~cm}$ on the radius of the I-band emission region. The smooth matter percentage in this lens is unconstrained.
\end{abstract}

The Manchester Microlensing Conference: The 12th International Conference and ANGLES Microlensing Workshop

January 21-25 2008

Manchester, UK

\footnotetext{
*Speaker.
} 


\section{Introduction}

Gravitational microlensing of multiply imaged quasars offers an excellent opportunity to constrain the size and geometry of emission regions in the source. This is traditionally done by observing and modelling microlensing-induced high-magnification events in lensed quasar lightcurves (e.g. [1], [16], [18]). Lensed quasars displaying anomalous flux ratios provide an alternative approach to this particular problem, which will be discussed here.

Lensing theory tells us that images closely straddling a critical curve should have approximately equivalent magnification [3], [4]. However, numerous lensed quasars are observed with one image in a close pair significantly demagnified with respect to the other. MG 0414+0534 is one such anomalous lensed quasar, for which Schechter and Moore reported an I-band flux ratio between images $A_{2}$ and $A_{1}$ of $0.45 \pm 0.06$ in observations taken on 1991 November 2-4 [13]. This observation will be used throughout the following analysis.

Microlensing offers a possible explanation for this discrepancy. Standard microlensing simulations, which assume that all matter in the lens is in the form of compact objects, yield an extremely low probability of 0.068 for a flux ratio lower than $A_{2} / A_{1}=0.45 \pm 0.06$ in MG 0414+0534 [15]. Adding a smooth matter component to the lensing galaxy in these simulations can significantly increase this probability, to values as large as 0.35 [14]. Other possible explanations for these anomalies exist. Millilensing by cold dark matter substructures is the most compelling alternative (e.g. [9]). However, millilensing should also affect the radio emission from the quasar, for which an anomalous flux ratio is not observed in MG 0414+0534 [7].

We have extended microlensing simulations presented elsewhere ([15], [14]) to include both the effects of varying source size and smooth matter component in the lens on the magnification distributions of images straddling a critical curve. We then use the anomalous flux ratio observed in MG 0414+0534 to place a constraint on the size of the I-band emission region in the source quasar.

A standard cosmology with $H_{0}=70 \mathrm{kms}^{-1} \mathrm{Mpc}^{-1}, \Omega_{m}=0.3$ and $\Omega_{\Lambda}=0.7$ is used throughout.

\section{Simulations}

We conducted microlensing simulations using an inverse ray-shooting technique (e.g. [8], [17]). The key parameters in these simulations are the convergence $\kappa_{t o t}$ (divided into a continuously distributed component $\kappa_{c}$ and a compact stellar component $\kappa_{*}$, following [14]) and the shear $\gamma$ of the lens at the image positions. These parameters for images $A_{1}$ and $A_{2}$ in MG 0414+0534 were taken from [15], and are provided in Table 1.

\begin{tabular}{lcccl}
\hline Image & Type & $\kappa_{\text {tot }}$ & $\gamma$ & $\mu_{\text {tot }}$ \\
\hline$A_{1}$ & minimum & 0.472 & 0.488 & 24.2 \\
$A_{2}$ & saddle & 0.485 & 0.550 & -26.8 \\
\hline
\end{tabular}

Table 1: Lensing parameters for the images of interest in MG $0414+0534\left(A_{1}\right.$ and $\left.A_{2},[15]\right)$.

Magnification maps covering an area of $24 \eta_{0} \times 24 \eta_{0}$ were generated, where $\eta_{0}$ is the Einstein Radius projected on to the source plane $\left(3.75 \times 10^{16}\left(M / M_{\odot}\right)^{1 / 2} \mathrm{~cm}\right.$ for MG 0414+0534). We allowed the smooth matter percentage in the lens to vary from $0 \%$ to $99 \%$. For each model, 

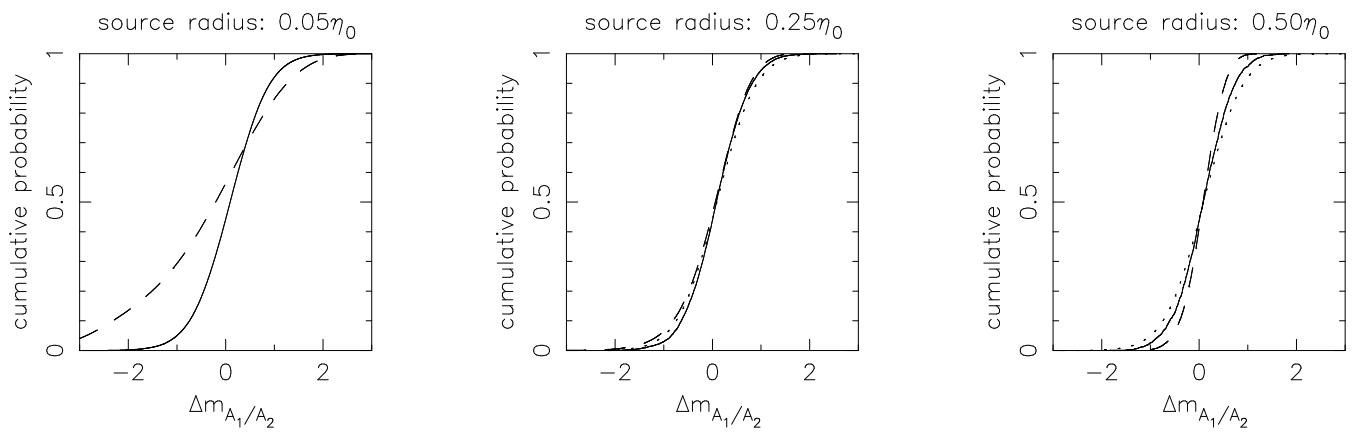

Figure 1: Cumulative probability distributions for $\Delta m$ between images $A_{1}$ and $A_{2}$ in MG 0414+0534. Smooth matter percentages of $0 \%$ (solid line) and $93 \%$ (dashed line) are displayed, for three characteristic source radii (left to right). The line representing the $0 \%$ smooth matter, $0.05 \eta_{0}$ source radius distribution is provided in the second and third panels (dotted line) for comparison.

20 magnification maps were generated, with a resolution of $2048 \times 2048$ pixels. This number of $24 \eta_{0} \times 24 \eta_{0}$ maps was found to provide enough statistically independent data points to construct reasonable probability distributions.

The magnification maps were then convolved with a Gaussian source brightness profile, with characteristic radius varying from $0.05 \eta_{0}$ to $2.00 \eta_{0}$. In physical units for MG $0414+0534$, this corresponds to a characteristic radius of $1.87 \times 10^{15}$ to $7.50 \times 10^{16}\left(M / M_{\odot}\right)^{1 / 2} \mathrm{~cm}$. A Gaussian source brightness profile is reasonable as [10] have shown that microlensing fluctuations are insensitive to all source model properties except the radius. Probability distributions for change in magnitude $\Delta m=2.5 \log _{10}\left(\mu_{2} / \mu_{1}\right)$ were constructed by dividing magnifications for image $A_{2}$ by those for image $A_{1}$ and binning the results.

\section{Flux ratio distributions}

In Figure 1 we provide simulated cumulative probability distributions for change in magnitude $\Delta m$ between images $A_{2}$ and $A_{1}$ in MG 0414+0534. In the first panel, we plot distributions for a source with characteristic radius $0.05 \eta_{0}$ (essentially a point source in our simulations) and a smooth matter component of $0 \%$ (solid line) and 93\% (dashed line). This illustrates the effect previously observed in [14]. For a small source, a flux ratio as low as $A_{2} / A_{1}=0.45$ (equivalently, $\Delta m=-0.87$ ) has a probability of 0.07 for the $0 \%$ smooth matter case, and 0.31 for the $93 \%$ smooth matter case.

When we increase the source radius to $0.25 \eta_{0}$ (Figure 1, panel two), the $0 \%$ and $93 \%$ smooth matter probability distributions become virtually identical. For larger source radii $\left(0.50 \eta_{0}\right.$ in panel 3 of Figure 1) the $93 \%$ smooth matter distribution is found to be narrower than the $0 \%$ smooth matter distribution. We therefore conclude that a large smooth matter component is only able to explain observed anomalous flux ratios if the source size is small relative to the Einstein Radius. Similar results were obtained in [6] for a different region of $\kappa-\gamma$ parameter space.

\section{Constraining the size of the emission region}

We now compare the anomalous observed flux ratio in MG $0414+0534 R_{o b s}=A_{2} / A_{1}=$ 


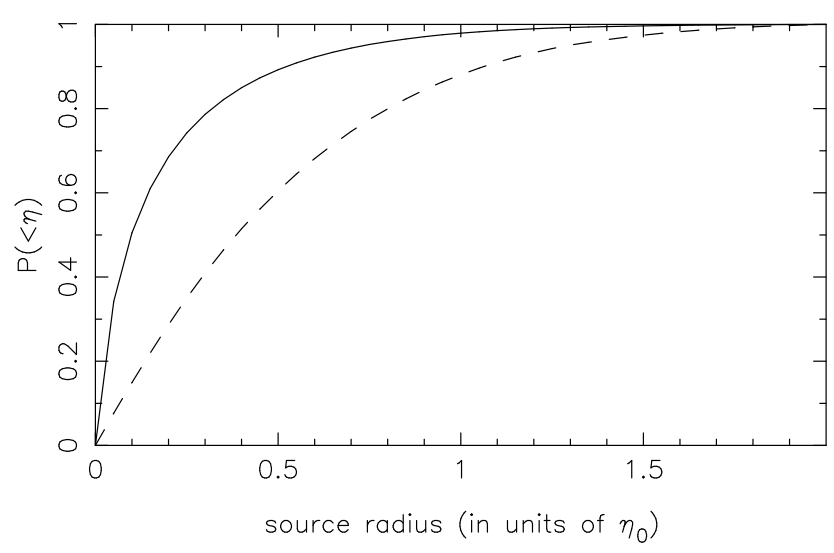

Figure 2: Cumulative probability that the I-band emission region in MG 0414+0534 is smaller than a radius $\eta$, given an observed flux ratio of $\left(A_{2} / A_{1}\right)_{o b s}=0.45 \pm 0.06$. Results are displayed for two source radius priors: logarithmic (solid line) and uniform (dashed line).

$0.45 \pm 0.06$ with our simulated conditional probability distributions for flux ratio. This allows us to construct likelihoods $L\left(R_{o b s} \mid s, \eta\right)$ for the observed flux ratio given source radius $\eta$ (in units of $\eta_{0}$ ) and smooth matter percentage $s=\kappa_{c} / \kappa_{\text {tot }}$. Using Bayes' theorem, we convert these likelihoods into an a posteriori differential probability distribution for smooth matter percentage and source size as a function of observed flux ratio:

$$
\left.\frac{d^{2} P}{d s d \eta}\right|_{R_{o b s}} \propto L\left(R_{o b s} \mid s, \eta\right) \frac{d P_{\text {prior }}}{d s} \frac{d P_{\text {prior }}}{d \eta}
$$

We then marginalise over the observed distribution for flux ratio, treating the error as a Gaussian with a characteristic radius equal to the observational error of \pm 0.06 ,

$$
\frac{d^{2} P}{d s d \eta}=\int d R\left(\left.\frac{d^{2} P}{d s d \eta}\right|_{R_{o b s}}\right) \frac{1}{\sqrt{2 \pi} \Delta R_{o b s}} \exp \left(\frac{-\left(R-R_{o b s}\right)^{2}}{2 \Delta R_{o b s}^{2}}\right)
$$

We used a constant Bayesian prior for smooth matter percentage, as the smooth matter percentage is a dimensionless quantity. A logarithmic Bayesian prior was chosen for source radius, as a prior which is flat in the logarithm ensures that the ratio of prior probability for two values of source size does not depend on the units chosen. We repeated our analysis using a constant Bayesian prior for source radius, in order to check the effect of prior selection on our results.

We then find marginalise over the smooth matter percentage,

$$
\frac{d P}{d \eta}=\int \frac{d^{2} P}{d s d \eta} d s
$$

to obtain the probability that the source is smaller than a particular radius,

$$
P(<\eta)=\int_{0}^{\eta} \frac{d P}{d \eta^{\prime}} d \eta^{\prime}
$$

The results of this analysis are provided in Figure 2, for both the logarithmic (solid line) and constant (dashed line) source radius priors. For the logarithmic prior, we find that the radius of 
the I-band emission region in MG $0414+0534$ is smaller than $0.70 \eta_{0}$ with a statistical confidence of $95 \%$. This corresponds to a physical radius of $2.62 \times 10^{16}\left(M / M_{\odot}\right)^{1 / 2}$. The constraint is less stringent where the constant source radius prior is used. This indicates that the range of source radii that are consistent with the data is considerable. The smooth matter percentage in this lens is unconstrained.

\section{Discussion}

The size limit obtained here is consistent with those determined for other lensed quasars, using different methods (e.g. [1], [16], [18]). We note, however, that those methods depend upon the apparent transverse velocity of the source, which is not well known. Our method is independent of this factor. Furthermore, we were able to obtain our limit using a single observation of the lensing system, whereas most earlier analyses have relied upon long-term monitoring.

MG 0414+0534 is not the only lensed quasar displaying anomalous flux ratios between close pairs of images. PG 1115+080, SDSS J0924+0219, WFI J2026-4536 and HS 0810+2254 are four other well-known examples [11]. These systems are being actively studied. Pooley and collaborators have investigated ten systems in the X-ray and optical and concluded that their optical emission regions are larger than those predicted by thin disk models by a factor of $\sim 3-30$ [12]. Chiba and collaborators are using mid-infrared and IFU observations of anomalous lensed quasars to place limits on the abundance of small-scale dark matter haloes on galaxy scales [5].

We have obtained contemporaneous observations of MG 0414+0534 across five filters using the $6.5 \mathrm{~m}$ Magellan telescopes, in collaboration with David Floyd. Theories of quasar accretion suggest that different wavelengths are emitted from different regions in the source. Current accretion disk models, however, have considerable difficulty accurately explaining observations [2]. Our data will allow us to probe the structure of the quasar central engine directly. The results of this analysis are forthcoming.

In conclusion, we find that microlensing simulations with a smooth matter component in the lens are able to explain anomalous flux ratios observed between lensed quasar images straddling a critical curve. The size of the emission region and the parity of the images are more significant factors than the smooth matter percentage in the lens. We place a $95 \%$ upper limit on the size of the I-band emission region in MG $0414+0534$ of $2.62 \times 10^{16}\left(M / M_{\odot}\right)^{1 / 2}$.

\section{References}

[1] T. Anguita, R. Schmidt, E. Turner, J. Wambsganss, R. Webster, K. Loomis, D. Long, R. McMillan, The multiple quasar Q2237+0305 under a microlensing caustic, A\&A 480 (2008) 327

[2] O. Blaes, Accretion Disks in AGNs, in proceedings of The Central Engine of Active Galactic Nuclei [astro-ph/0703589]

[3] R. Blandford, R. Narayan, Fermat's principle, caustics, and the classification of gravitational lens images, ApJ 310 (1986) 568

[4] K. Chang, S. Refsdal, Flux variations of QSO 0957+561 A, B and image splitting by stars near the light path, Nature 291 (1979) 620 
[5] M. Chiba, T. Minezaki, K. Inoue, N. Kashikawa, H. Kataza, H. Sugai, Dark Matter Substructure in Lensing Galaxies, to appear in proceedings of Panoramic Views of Galaxy Formation and Evolution [astro-ph/0804.0282]

[6] A. Congdon, C. Keeton, S. Osmer, Microlensing of an extended source by a power-law mass distribution, MNRAS 376 (2007) 434

[7] C. Katz, J. Hewitt, Further radio investigations of gravitational lensing in MG 0414+0534, ApJ 409 (1993) L9

[8] R. Kayser, S. Refsdal, R. Stabell, Astrophysical applications of gravitational micro-lensing, A\&A 166 (1986) 36

[9] C. Kochanek, N. Dalal, Tests for substructure in gravitational lenses, ApJ 610 (2004) 69

[10] M. Mortonson, P. Schechter, J. Wambsganss, Size Is Everything: Universal Features of Quasar Microlensing with Extended Sources, ApJ 628594

[11] D. Pooley, J. Blackburne, S. Rappaport, P. Schechter, W. Fong, A Strong X-Ray Flux Ratio Anomaly in the Quadruply Lensed Quasar PG 1115+080, ApJ 648 (2006), 67

[12] D. Pooley, J. Blackburne, S. Rappaport, P. Schechter, X-Ray and Optical Flux Ratio Anomalies in Quadruply Lensed Quasars. I. Zooming in on Quasar Emission Regions, ApJ 661 (2007) 19

[13] P. Schechter, C. Moore, The lensing Galaxy in MG 0414 + 0534, AJ 105 (1993) 1

[14] P. Schechter, J. Wambsganss, Quasar Microlensing at High Magnification and the Role of Dark Matter: Enhanced Fluctuations and Suppressed Saddle Points , ApJ 580 (2002) 685

[15] H. Witt, S. Mao, P. Schechter, On the universality of microlensing in quadruple gravitational lenses, ApJ 443 (1993) 18

[16] J. Wambsganss, B. Paczynski, P. Schneider, Interpretation of the microlensing event in QSO $2237+$ 0305, ApJ 358 (1990) L33

[17] J. Wambsganss, B. Paczynski, N. Katz, A microlensing model for QSO 2237 + 0305, ApJ 352 (1990) 407

[18] J. Wyithe, R. Webster, E. Turner, D. Mortlock, A gravitational microlensing determination of continuum source size in Q2237+0305, MNRAS 315 (2000) 62 